\title{
CONTEXTUAL ANALYSIS AS SUPPORT FOR SUCCESSFUL INNOVATION IN COMPLEX ENVIRONMENTS
}

\author{
Peter M. Bednar \\ University of Portsmouth \\ Portsmouth, UK \\ Christine Welch \\ University of Portsmouth \\ Portsmouth, UK
}

\begin{abstract}
In order to survive and thrive, organizations need to adapt, reinvent themselves, and innovate. However, many intentional efforts to bring about innovation do not succeed. In order to be successful, innovation requires the support of individuals throughout the environment in which it is emerging. In many cases, such support is not forthcoming for a variety of reasons. In this paper, the authors discuss a number of barriers that may inhibit the success of innovative practice. They then discuss a role for contextual analysis as the means to target individual engagement, and present an example of a method which could be used to conduct such analysis.
\end{abstract}

\section{INTRODUCTION}

Innovation is associated with invention of novel artefacts; during the $1980 \mathrm{~s}$, mobile cellphones were a significant innovation. However, the Oxford Dictionary of English defines innovation as "a new method, idea, product, etc." Technological innovations do not just relate to creation and exploitation of physical artefacts, but include behavioral, social, or thought processes - ways people in organizations think about things, or ways in which they carry out the organization's work. An example appears in Ciborra's (1993)

Please use the following format when citing this chapter:

Bednar, P., and Welch, C., 2007, in IFIP International Federation for Information Processing, Volume 235, Organizational Dynamics of Technology-Based Innovation: Diversifying the Research Agenda, eds. McMaster, T., Wastell, D., Ferneley, E., and DeGross, J. (Boston: Springer), pp. 149-161. 
discussion of design in relation to strategic information systems. Drawing on a number of cases studied, he points to difficulties in exploiting the full innovative potential of new developments due to unexpected consequences: "events, behaviors and features of systems and the people who use them fall outside the scope of original plans and specifications" (p. 178). He suggests these systems are not so much designed as emergentearly adopters recognizing features that were initially overlooked or unplanned, but prove to be useful. In the second millennium, the "mobile" phone is surrounded by innovation, as much socio-cultural and fashion-oriented as technical, if not more so. Innovation-inuse is in focus here. Innovation involves creation of new information about resources, goals, processes, etc., and the existing stores of skill and competence residing in an organization represent at the same time potential for successful innovation and a constraint upon it (Pralahad and Hamel 1990).

Ciborra reminds us that there is always a knowledge gap to be addressed when tackling innovation, and that there are only two ways to bridge it: by incremental muddling-through (bricolage), or by radical learning processes which confront existing routines and core competences. The latter may be painful for individuals as well as organizations, as it involves moving out of a zone of familiarity to confront uncertainty (Ciborra 1993). Rogers (2003), in his well-known work on diffusion of innovation, highlights a number of attributes which appear to influence the rate of adoption. These include complexity (i.e., individual perceptions of how easy the novelty is to understand and use), trialability (i.e., the degree to which individuals have an opportunity to experiment on a risk-limited basis), and observability (i.e., the degree to which results of innovation can be viewed by others and described). We suggest that all of this may be resolved into one issue, which impacts upon the success of technological innovation, and this is the way in which individuals, and the organization in which they are located, deal with uncertainty. Michael (1985), cited in Schein (1992), refers to empirical evidence showing that managers' ability to tolerate uncertainty may be of great importance to organizational success during disturbance and change. People often react to conditions of uncertainty by seeking ways to simplify their problem-space. They may attempt to break down a complex problem into smaller ones, or make assumptions that ready solutions must exist if only they can be "found." Indeed, complexity is a phenomenon that arises through perceptions of actors involved in a scenario, who choose to regard it as complex. Checkland (1999) reflects that his early work with the soft systems methodology involved a movement away from perception of obvious problems to be solved, toward a perspective that there were situations regarded by some people as problematic.

Organizational problems, particularly where new initiatives are considered, are experienced as unstructured. Successful implementation of innovation is an inherently political process (Argyris 1990; Weick and Sutcliffe 2002). Weick (1995) discusses ways in which individuals transform a perceived problem-space that is unstructured (uncertain) into a structure d uncertainty, through sense-making processes. In attempting to make sense of an unstructured problem, people try to move from a position of not knowing what to do, to a position of knowledge about a range of alternative possibilities from which a solution might be created (i.e., ambiguity). Unfortunately, organizational decision making is always subject to pressure of time. In many instances, this can lead to a desire to reach an early resolution of uncertainty, and thus to over-simplification of complex problem-spaces. A rush to early consensus on design or implementation of innovation may inhibit the process of emergence to which Ciborra (1993) refers. 
In this paper, the term contextual analysis is used to mean inquiry into a number of contextual dependencies experienced by actors contemplating innovation/change. Examples of contextual dependencies include experiences of work roles, of political dimensions in the organization, or even of physical aspects of the work environment. The authors believe contextual inquiry to be useful in efforts to bring about beneficial change in human activity systems. Its benefit lies in the way in which it addresses the nature of transformation processes, and it may be conducted before, during, and after actions for change. Contextual inquiry may be useful in any environment actors choose to treat as complex. Traditionally, in scientific investigation, perception of complexity depends upon the qualities of the instrument used (e.g., a drop of water appears very simple until it is examined under a microscope, at which point the complex array of biological and chemical constituents contained within it emerges). We suggest contextual inquiry as an instrument enabling emergence of complexity in organizational situations. The authors agree with a widespread view (see Argyris 1990; Bateson 1972; Nissen 2007) that individuals need to become engaged with a process of innovation in order to support it successfully. We believe that collections of inquiries into multiple levels of specific contextual dependencies may lead to emergence of insights which provide support for engagement.

In the next section of this paper, the authors discuss barriers which many people have identified as inhibitors of successful innovation. Here, they are addressed together as a collective of contextual dependencies. We suggest how contextual analysis provides a key to overcoming them, and hence supports organizational members to bring about a necessary transformation of uncertainty into ambiguity. The following section outlines one method for contextual inquiry (the strategic systemic thinking framework), which supports individuals to explore these contextual dependencies and create new perspectives on innovation and change.

\section{BARRIERS TO SUCCESSFUL INNOVATION}

C. West Churchman (1971) has suggested that an inquiring system has the capability of asking itself what would be the characteristics of an inquiring system that did not function in accordance with the tenets of its own design. It is recognized in cybernetics that every distinct dimension of a complex system needs to be controlled in a way which is appropriate to its characteristics. This is Ashby's (1964) law of requisite variety. By analogy, every dimension of a complex problem space needs to be addressed with appropriate analytical approaches. We do not necessarily need a multiplicity of tools and techniques, catering for many different threads of problem spaces. What is required is exercise of human ingenuity to reflect and to adapt those methods available to us in appropriate ways (Ciborra 2002). We consider that individual engagement with innovative practice may be supported by methods that explore contextual dependencies. However, analysts wishing to adopt such methods first need to contemplate aspects of the problem space surrounding innovation. Since the problem space is unknown and uncertain in its boundaries, it is unlikely that a predefined archetype of method (which assumes a known problem) would suffice in itself without adaptation. Therefore, analysts need to develop an approach that specifically targets the needs of individuals from a particular inquiry, in a particular context. This approach needs to be designed to 
address those barriers to innovation which the analysts' preliminary investigations suggest to be present.

In business organizations, consultation can take place among stakeholders about initiatives that are planned. The question arises how far this is comprehensive, or represents a true attempt to establish dialogue, and how far the resultant opinions actually inform decisions about innovation. Some authorities (e.g., Argyris and Schön 1978, 1996) appear to suggest that individuals' views within an organizational setting are not always expressed effectively, nor are they necessarily acted upon. Change in behavior is much more difficult to achieve than mere descriptions of change. There is often a difference between our descriptions of what we think we do (what Argyris and Schön have called espoused theories) and what others can perceive that we do (our theories-inuse) (Argyris and Schön 1978). People do not always perceive or admit to this difference themselves. Efforts are required to surface these problematic issues in the form of inquiry into the informal and unconscious behavior of individuals (their intra-individual contexts) and the interactions between individuals (their interindividual contexts). The word communication could describe either a one-way process in which a message travels from sender to receiver, or a two-way process in which the receiver responds by providing feedback (see Grunig 1992). Communication processes might be symmetrical, reflecting equal degrees of engagement by the participants, or asymmetrical where one party is engaged or empowered to a greater degree than the other. Effective consultation on change requires a genuine engagement by both parties in a constructive and open dialogue. We believe that methods for inquiry are needed which provide specific support for creation of such a dialogue.

Two-way communication can provide for feedback between the parties. We frequently experience asymmetrical, two-way communication during political campaigns. Attempts may be made to persuade voters to a particular point of view through persuasive speeches. Sometimes "inconvenient" facts will be omitted or distorted in an attempt to engineer the goodwill of voters. In the course of the campaign, there may be opportunities for feedback, either directly (e.g., through hustings), or indirectly (through opinion polls). In contrast, two-way symmetrical communication would ideally involve dialogue between parties, who engage in an effort to interpret one another's contributions in order to promote a meeting of minds. Perfect symmetry in communication may be impossible to achieve. However, a participant in dialogue may perceive it to be more or less symmetrical. Each party could make an effort to put forward a truthful view, listen to the response of the other, and proceed to a discussion of areas of disagreement. Argyris (1990) has argued that the process of symmetrical two-way communication is a basic foundation which is a prerequisite for a learning organization to be built or sustained. We view symmetrical communication as a continuously changing process of inquiry, which is contextually dependent. Some aspects of contextual dependency are further developed later in this paper.

If managers are to promote support for a productive learning cycle in their organization, in relation to innovation, then individuals need to be motivated and empowered to think and express themselves, and to collaborate and share their ideas. Managers need to recognize a possibility that individuals could use their creative powers in ways that undermine such aspirations, or subvert them to other ends. Argyris (2004) draws attention to defensive strategies, which are sometimes adopted in organizations where conditions of great uncertainty are experienced. He points to an example from an 
empirical study by Van de Ven and Polley (1992), where colleagues pursued collusive action strategies of impression management. Here, members of a project team, together with resource managers, actively avoided recognizing errors and difficulties, so as to avoid embarrassment arising from the challenge of sense-making. One impact of these tactics was, of course, to inhibit opportunities for learning through trial and error, and reflection. Argyris highlights for us an ethical double bind, which he perceives to be involved. These individuals colluded in order to cover up disagreeable truths through feelings of loyalty to their colleagues and their organization, while attempting to show integrity by not exposing this process. We can turn to the work of Gregory Bateson (1972) for further illumination of situations where individuals are caught in a double bind. As conscious human beings, we have no choice but to reflect on our experience. The question for us all to address, however, is the form this reflection takes. Defensive strategies may tend to perpetuate the double bind in which individuals are caught. Bateson has suggested that an individual may need to adopt an observer perspective to enable herself to break out from such entrapment.

Organizational knowledge may not be distributed in a homogeneous way. Seely Brown and Duguid (2002), for instance, point out how communities of practice emerge within organizations. While organizations may develop a distinctive culture over time, it is also likely that there will be smaller groups within the organization who have more interests in common with each other than with colleagues generally. These clusters of individuals develop distinctive values and norms of their own. Sometimes, such a community within a particular organization finds more common ground with a similar community in the outside world than with their own colleagues. These authors point to examples where organizational knowledge has proved to be both "sticky" and "leaky" when it relates to innovation. If the prevailing paradigm by which members of a company make sense of their environment is nonconducive to inventions, those with creative ideas may be forced to set themselves apart. Their ideas do not then permeate the organization freely their knowledge is sticky. However, through networks of practice they develop across organizational boundaries, ideas may leak from the organization to more receptive ground elsewhere (for discussion of Xerox PARC's failure to exploit GUI, which was subsequently taken up by Apple, see Seely Brown and Duguid 2002, p. 159).

What is clear from this is that managers' ability to develop and support an environment that promotes organizational learning, and helps to promote paradigm shift (Kuhn 1970), may be a key to success. It has been suggested that organizations can become "psychic prisons" in which individuals experience entrapment within their own constructed realities (Morgan 1997). Such entrapment prevents individuals and groups from espousing new knowledge if it conflicts with established and familiar patterns. Weick and Sutcliffe (2002) reinforce this when they suggest a need to promote a collective state of "mindfulness." More specifically, they suggest a need for on-going attention to existing expectations in the light of new experiences, and, simultaneously, engagement with creation of new expectations. A state of mindfulness would be characterized by a desire by individuals and groups to learn from their mistakes, a focus on disconfirming, rather than confirming behavior, and a respect for uncertainty. There would be a prevailing recognition that knowledge has limits, and that professional behavior and competence depend upon making explicit consideration of those boundaries.

An organization that successfully encourages a creative culture of mindfulness may be regarded as one that is able to support a constructive dialectic, between meaningful 
use of innovation and reflection upon that use. In a dialectical relationship between use and reflection on use, each element is subject to change. Reflection triggers change in use, and such change triggers further reflection. Lived human experience, and reflection upon that experience, seems to shape a double helix (further discussion of this double helix metaphor may be found in Nissen 2007). It is suggested that failures of innovative projects come about through lack of support for individuals and groups to engage in learning. We use the term failure here to mean a problematized difference between intended consequences and unintended (but recognized) consequences of design and action, from a specific observer's point of view (Markus and Robey 2004). This last point we consider to be most important because it highlights a further requirement for learning to take place. This is a desire to engage in learning activities (Bednar and Welch 2006). What incentive is required to induce individuals to espouse learning for change? What benefits would they derive and how might they become aware of these benefits? If intrinsic benefits to individuals are not apparent, can extrinsic inducements be appropriate? Desire for innovation may exist, but how is it to be articulated, assessed, and acted upon within the organizational context? If motivation is a necessary condition for adoption, then learning for meaningful use is an equally important aspect of socialization. In the words of Chris Argyris,

It is not possible for human beings to engage de novo the full complexity of the environment in which they exist. Life would pass them by. Human beings deal with the challenge by constructing theories of action that they can use to act in concrete situations (2004, p. 8).

When an attempt is made to evaluate effectiveness in managing or designing organizational change, concepts of analysis become important. Good practice requires an understanding that addresses intrinsic and contextually dependent characteristics of organizational activities. An understanding can only come about through relevant evaluative and analytical strategies (Avgerou and Madon 2004). Evaluation can be described as a result of both inquiring and reflecting thought processes (i.e., mental activity intrinsically dependent upon a demonstrated, contextually dependent desire to explore a certain problem space). We see anaysis as an inquiry into the assumed-to-be unknown and/or a questioning of the assumed-to-be known. Evaluation, we suggest, is a consolidating process, where judgments are made, and assumed truths and knowledge are incorporated into some kind of hierarchy. Together, an analysis (i.e., creation of new knowledge) and evaluation (i.e., categorization of existing knowledge) represent closing of a learning circle. Any conscious reflection over the requirements for a higher quality learning circle could become a daunting exercise as it involves raising the quality of knowing. The concept of the learning organization, presented by Argyris and Schön $(1978,1996)$ is suggested as a means to reflect upon and reevaluate knowledge created by individuals within an organizational context. At this point, it is useful to consider the possibility of multiple orders of learning (see, for example, the discussion by Bateson 1972, p. 287). These are described by Argyris and Schön (1978) in terms of single or double loops. When an individual needs to solve an immediate problem (i.e., close a perceived gap between expected and actual experience), she may harness her sensemaking processes within a context of existing goals, values, plans, and rules, without questioning their appropriateness. However, if she goes beyond this to challenge 
received wisdom and to critically appraise the assumptions previously applied, doubleloop learning occurs. We view the resulting process as creating a productive learning spiral, and consider this to be essential to successful organizational innovation.

\section{CONTEXTUAL ANALYSIS}

We consider contextual analysis to offer a key to unlocking many of the barriers outlined above. Methods for analysis that specifically support exploration of contextual dependencies could lead to creation of alternative perspectives on innovation. The impact of innovation can be very significant for organizational development. Therefore, a high standard of analysis, uncovering needs and expectations that members of the organization have from the proposed innovation, is crucial. For example, evidence exists to suggest that an effective inquiry into the fit between information systems processes and business processes in a specific organization could make or break the business (Markus and Robey 2004). While any expenditure of resources faces an opportunity cost, we believe that time and money spent in promoting effective inquiry will be justified. The importance of organizational analysis, to make sense of possible business process enhancements through application of new ideas, routines, or technologies, cannot be overemphasized. Implementation practices have important consequences for the political and social systems within organizations.

One relevant example is Nestlé's attempt to introduce enterprise resource planning (ERP) systems in the late 1990s/early 2000s (see Worthen 2002). A clear business case could be made for systems to enable the company to act as one entity worldwide, and thus make cost efficiencies and manipulate its market power to greater effect. However, attempts to introduce SAP systems based on best practice in business processes were initially perceived by management as unsuccessful. Reflection by management highlighted lack of involvement of key stakeholders and concentration on technical matters as contributing factors. An independent analyst, whose assessment of the company potentially affected its share price, commented that the project "touched the corporate culture" and was thus a risk. Ultimately, the project was recommenced from scratch with a more inclusive approach. This case illustrates that, if inquiries conducted prior to, and during, introduction of an innovation are confined to a superficial examinations of goals, tasks, and decisions, the results may be very unsatisfactory. Successful implementation depends upon effective inquiry into a multitude of issues in the organizational arena forming the backdrop to any novel development (see Walsham 1993).

In order to overcome the problem of sticky knowledge relating to innovation, outlined by Seely Brown and Duguid (2002), everyone concerned in the organizational context requires enhanced understanding of the problem space. Who is supposed to create a better understanding, on behalf of whom, how, and of what? Experience suggests that expert-dominated (and/or management-imposed) solutions to organizational problems are not always bought into by the actors. This has led researchers to consider participative methods for engaging actors in creative processes. Examples can be seen in the field of information systems development, for example client-led Design (Stowell and West 1994) or the ETHICS methodology (Mumford 1994). In this type of approach, a focus is placed on a communication gap. It could be assumed that users simply do not have sufficient awareness of their own needs to be able to communicate them effectively 
to an analyst, and that if techniques can be found to bridge this gap, then all will be well. We argue that, while efforts to overcome the communication gap are worthwhile, this view still fails to address contextually dependent dimensions of complex organizational problem spaces. It is not simply that the users are unable to articulate their preexisting requirements, and therefore need a developed language and tools. First, they must be able to create an understanding of what those requirements might be, in relation to a problem space that represents their experience of working life. From our point of view, support for this creative process is vital to any vision of successful innovative practice. Furthermore, we believe that participation by actors is not sufficient; a genuinely collaborative approach is needed, in which actors own and control the inquiry for themselves. Analytical efforts must continue throughout the process of change. Those individuals in whose environments innovative artefacts, processes, or methods will reside must own and control the analysis, supported by professional facilitators, in order to be able to explore their understandings of their experiences.

The inherent complexities of organizational life, and the uncertainty associated with organizational contexts, lead us to highlight epistemological problems for a foundational view of knowledge. Opinions differ about the meaning of the term knowledge. As we focus attention on processes of knowledge creation and sharing, it is useful to consider this. A discussion is offered by Davenport and Prusak.

Knowledge is a fluid mix of framed experience, values, contextual information, expert insight and grounded intuition that provides an environment and framework for evaluating and incorporating new experiences and information. It originates and is applied in the minds of knowers. In organizations, it often becomes embedded not only in documents or repositories but also in organizational routines, processes, practices and norms (Davenport and Prusak 2000, p. 5).

We view contextual analysis as an approach that addresses such problems and their implications for research into organizational systems. An analyst taking a relative view of knowledge needs to look critically at a series of exemplary approaches, which use different ontologies and epistemologies. For example, Information Systems research relating to contextual dependencies attempts, inter alia, to build on previous core research in information systems (e.g., by exploring ways in which contemporary open systems thinking can be applied to specific critical issues) (see Maturana and Varela 1980; Nissen 2007). Particular emphasis is placed on multiple sense-making processes, and ways in which these are played out within the frameworks of learning organizations. Some researchers have focused on individual or group managerial perspectives, such as where a business manager is a user (Carlsson 1993; Ciborra 1993). More recently, some efforts to deal with context have involved the use of actor-network theory (Latour 1999). Contextual dependency as a concept is relevant here because it supports a focus of inquiry on unique individuals, and their beliefs, thoughts, and actions, in specific situations and contexts. This kind of inquiry is intended to provide support to individuals in a contextually dependent creation of necessary knowledge. This in turn enables successful communication, analysis, evaluation, and, eventually, implementation of innovation.

We do not suggest that contextual analysis should necessarily replace other, traditional approaches to innovation. It is a complementary approach which may help those 
involved to avoid a conflict related to unproblematic assumptions of ontological beliefs and logical empiricism. For example, analysts sometimes hold unquestioned beliefs of unproblematic objectivity and truths. Like some of the traditional communicational theories, traditional approaches to organizational analysis may be based on assumptions around a sender-receiver model (see the earlier discussion). A contextual approach to analysis is intended to focus instead on individual perspectives. Thus an inquiry based on contextual analysis would not ask what an organization wants to achieve. Instead, it would support the actors to ask what they themselves want to achieve in the organizational context. For instance, they would question what roles and specific purposes their activities have; what makes their unique situations recognizable within the organizational problem space. The focus of inquiry is, therefore, individual assumptions and needs within the space of an open organizational system from the actors' own points of view. We describe this as a bottom up perspective on innovation. Contextual analysis, as an approach which tries to take contextual dependencies into consideration, may represent a response to escalation in complexity in organizational life.

In our view, approaches to analysis depend in practice on ways in which a problem space is framed, and by whom. This principle has been given thorough consideration by Ulrich (2001), in his extensive discussion of boundary critique. In soft systems methodology (Checkland 1999), for example, a consideration of individual Weltanschauungen (or world views) is required of those attempting to explore complex problem space. (For a contemporary discussion of the role of Weltanschauungen in analyses, see BergvallKăreborn 2002.) With the help of analysis relating to narratives of mental constructs, relationships can be discussed within a more context-dependent framework of reasoning. One such approach is discussed next.

The strategic systemic thinking framework (Bednar 2000) aims to apply specially adapted methods to investigate how people construct understanding and meaning, and how need for knowledge, and new knowledge, are created by individuals within this process. The framework was originally developed through one of the author's experiences over a number of years in industry in dealing with complex problem spaces. Discussions later took place following a series of workshops for managers on systemic methods. These led to an initial view of a framework for contextual inquiry which has been developed over a number of years of further research. Basing inquiry in hermeneutic dialectics involves recognition of uncertainties and ambiguities inherent in socially constructed views of human activity. This type of inquiry seeks for transparency as well as clarity in investigations, in order to emphasize the self-awareness of individuals. Efforts to achieve precision, rather than transparency, involve a danger of separation between observations made and the unique perspectives of observer and observed (e.g., Maturana and Varela 1980; Nissen 2007). The intention behind SST is to promote inquiry into organizational knowledge creation and sharing, which avoids what Radnitzky (1970) describes as an artificial separation of theory and practice.

There are three aspects of inquiry using SST-intra-analysis, inter-analysis, and value analysis - which may be undertaken in any order and are iterative. Intra-analysis supports individuals in exploring their own perspectives on the perceived situation. A variety of methods such as rich pictures or brainstorming are used to support exploration of individual sense-making processes, and creation of individual narratives. Interanalysis provides a vehicle for groups of people to communicate their individual narratives to one another, and attempt to create an understanding of each one. Members 
of the group then discuss one another's narratives in order to create a view of the range of different alternative opinions expressed in relation to the problem space. The purpose here is to create an enriched dialogue among actors without seeking a premature

consensus. Value analysis, an examination of the known, inquires into what has been learned from intra/inter-analyses and attempts to relate it to a frame of reference. Actors conduct an examination of values influencing and constraining the analyses, consider prioritization from political and cultural perspectives, and assess risks. The overall purpose of SST is to support inquiry into multiple levels of contextual dependencies, in order to enable creation of new knowledge, communication of new perspectives, consolidation, and evaluation.

\section{CONCLUSIONS}

Contextual inquiry takes into account the messiness of everyday experience, and individuals' attempts to search for meaning through sense-making activity. Some approaches to organizational analysis are intended to simplify problem spaces, in a desire to steer a manageable path through rich and diverse sources. However, the authors of this paper propose instead a structured, systemic "complexification" through inquiry into contextual dependencies. Such an inquiry draws upon unique individual beliefs, actions, and perspectives in specific organizational situations, and the living history of an organizational group from each individual's point of view. However, it must be noted that we consider that methods seeking precision and clarity and those seeking transparency may be regarded as complementary to one another.

Processes through which people create and recreate their knowing are at once deeply personal, contextual and social. Knowing comes about through perception of change. This draws upon Bateson's (1972) idea of a "difference that makes a difference." Seely Brown and Duguid reflect on this point as follows:

The background has to be in place for the information to register. The forces that shape the background are, rather, the tectonic social forces, always at work, within which and against which individuals configure their identity. These create not only grounds for reception, but grounds for interpretation, judgment, and understanding (2002, p. 139).

The contextually dependent nature of any problem description, including meaning and defined purpose, means that responsibility for understanding, sense-making, and creation of a problem redefinition cannot be delegated to an expert (or devolved upon any technique or technology). Every individual will perceive purpose and relevance from her/his own unique perspective, and must therefore take ownership of the problem space and be her/his own analyst. However, means are needed to support and facilitate people in their contextual inquiries, sponsored by management. This must then, by definition, involve some delegation. There is a paradox for us here, in that each individual's espoused theories and theories-in-use will be distinctive and cannot be resolved into a simple consensus (Argyris and Schön 1978). Therefore, in order to bring about a supportive environment for purposeful change, we need a recognition that this resolution cannot be achieved. 
We have pointed to a number of factors which contribute to success or failure in making sense of, and responding to, uncertainty in complex problem spaces. We have demonstrated that a number of barriers exist within organizational environments which inhibit successful innovation and change. Our conclusion is that support for successful innovation in complex environments requires commitment from the main organizational protagonists. Promotion of commitment for change is in itself a political agenda, as recognized by both Argyris (1990) and Weick (Weick 1995; Weick and Sutcliffe 2002). An essential factor in the success of innovative projects may be a combination of individual and group sense-making processes which require support through constructive dialogue. We argue here that there is a need for managers to promote a culture of mindfulness so that a learning organization may be created. In an environment where uncertainty is embraced, rather than feared, and second order learning is fostered, successful implementation of innovation may be supported.

\section{References}

Argyris, C. Overcoming Organizational Defenses: facilitating Organizational Learning, Englewood Cliffs, NJ: Prentice Hall, 1990.

Argyris, C. Reasons and Rationalizations: The Limits to Organizational Knowledge, New York: Oxford University Press, 2004.

Argyris, C., and Schön D. A. Organizational Learning, Reading, MA: Addison Wesley, 1978.

Argyris, C., and Schön D. A. Organizational Learning II: Theory, Method and Practice, Reading, MA: Addison Wesley, 1996.

Ashby, W. R. An Introduction to Cybernetics, London: Methuen, 1964.

Avgerou, C, and Madon, S "Framing IS Studies: Understanding the Social Context of IS Innovation," in C. Avgerou, C Ciborra, and F. Land (eds.), The Social Study of Information and Communication Technology: Innovation, Actors, and Contexts, New York: Oxford University Press, 2004, pp 162-182.

Bateson, G. Steps to an Ecology of Mind, Part III, Chicago: University of Chicago Press, 1972.

Bednar, P. M. "A Contextual Integration of Individual and Organizational Learning Perspectives as Part of IS Analysis," Informing Science (3:3), 2000.

Bednar, P. M., and Welch, C. "Incentive and Desire: Covering a Missing Category," in Proceedings of Mediterranean Conference on Information Systems: A Comparative Distinction of Mediterranean Information Systems, San Servolo, Venice, Italy, October 5-9, 2006, pp. 53-61.

Bergvall-Kåreborn, B. "Enriching the Model-Building Phase of Soft Systems Methodology," Systems Research and Behavioral Science (19:1), 2002, pp. 27-48.

Carlsson, S. A Longitudinal Study of User Developed Decision Support Systems, Ph.D. Thesis, Department of Informatics, Lund University, Sweden, 1993.

Churchman C. W. The Design of Inquiring Systems: Basic Concepts of Systems and Organizations, New York: Basic Books, 1971.

Checkland, P. Systems Thinking, Systems Practice: A 30-Year retrospective. Chichester, England: Wiley, 1999.

Ciborra, C. U. The Labyrinths of Information: Challenging the Wisdom of Systems, Oxford, England: Oxford University Press, 2002.

Ciborra, C. U. Teams, Markets and Systems: Business Innovation and Information Technology, Cambridge, England: Cambridge University Press, 1993.

Davenport, T. H., and Prusak, L. Working Knowledge: How Organizations Manage What They Know, Boston: Harvard Business School Press, 2000. 
Grunig, J E Excellence in Public Relations and Communication Management, Mahwah, NJ: Lawrence Erlbaum Associates, 1992.

Kuhn, T. S. The Structure of Scientific Revolutions, Chicago: University of Chicago Press, 1970.

Latour, B. Pandora's Hope: Essays on the Reality of Science Studies, Cambridge, MA: Harvard University Press, 1970.

Markus, M. L., and Robey, D. "Why Stuff Happens: Explaining the Unintended Consequences of Using IT," in K. V. Andersen and M. T. Vendelo (eds.), The Past and Future of Information Systems, New York: Elsevier, 2004, pp. 61-93.

Maturana, H., and Varela, F. Autopoeisis and Cognition, Dordrecht, Holland: D.Reidel Publishing, 1980.

Michael, D. N. On Learning to Plan and Planning to Learn, San Francisco: Jossey-Bass, 1985.

Morgan, G. Images of Organization, Thousand Oaks, CA: Sage Publications, 1997.

Mumford, E. Systems Design: Ethical Tools for Ethical Change, Baskingstoke, England: Macmillan Press, 1996.

Nissen, H-E. "Beyond Double Helix Relationships to Understand and Change Informing Systems," Informing Science Journal, Special Issue: A Double Helix Relationship of Use and Redesign in IS?, 2007 (forthcoming).

Pearsall, J., and Hanks, P. (eds.). The Oxford Dictionary of English ( $2^{\text {nd }}$ revised edition), Oxford: Oxford University Press, 2005

Pralahad, C. K., and Hamel, G. "The Core Competences of the Corporation," Harvard Business Review (68:3), May-June 1990, pp. 79-93

Radnitzky, G. Contemporary Schools of Metascience, Göteborg, Sweden: Akademiforlaget, 1970.

Rogers, E. M. Diffusion of Innovation ( $5^{\text {th }}$ ed.), New York: Free Press, 2003.

Schein, E. H. Organizational Culture and Leadership ( $2^{\text {nd }}$ ed.), San Francisco: Jossey-Bass, 1992.

Seely Brown, J., and Duguid, P. The Social Life of Information, Boston: Harvard Business School Press, 2002.

Stowell, F., and West, D. Client-Led Design: A Systemic APproach to Information Systems Definition, London: McGraw-Hill, 1994.

Ulrich, W. "Critically Systemic Discourse: A Discursive Approach to Reflective Practice in ISD, Parts 1 and 3," The Joumal of Information Technology Theory and Application (3:3), 2001, pp. 55-106.

Van de Ven, A. H., and Polley, D. "Learning While Innovating," Organization Science (3:2), February 1992, pp. 92-116.

Walsham, G. Interpreting Information Systems in Organizations, Chichester, England: Wiley. 1993.

Weick, K. Sense-Making in Organizations, Thousand Oaks, CA: Sage Publicatoins, 1995.

Weick, K., and Sutcliffe, K. Managing the Unexpected, San Francisco: Jossey-Bass, 2002.

Worthen, B. "Nestlé's ERP Odyssey," CIO Magazine, May 15, 2002 (available online at http://www.cio.com/archive/051502/nestle.html).

\section{About the Authors}

Peter M. Bednar is originally from an engineering background and has several years of relevant industrial experience. Since 1997, he has been working as an academic. His research covers contextual analysis, organizational change, and information systems development, and he has published several book chapters and many articles in these fields. He is currently a senior lecturer in the School of Computing at the University of Portsmouth, UK, and is also affiliated with the Department of Informatics at Lund University, Sweden. He can be reached by e-mail at peter.bednar@ics.lu.se. 
Christine Welch is a principal lecturer in the Department of Strategy and Business Systems, part of the Business School at the University of Portsmouth, UK. Her research interests include critical system thinking, contextual analysis, and knowledge management. She has published a number of articles in these fields. Christine is also a member of the board of the UK System Society. She can be reached by e-mail at christine.welch@port.ac.uk. 